\title{
Structural and Gelation Characteristics of Metallo-Supramolecular Pol- ymer Model-Network Hydrogels Probed by Static and Dynamic Light Scattering
}

\author{
Martha Franziska Koziol, Karl Fischer, Sebastian Seiffert* \\ Department of Chemistry, Johannes Gutenberg-Universität Mainz, Duesbergweg 10-14, D-55128 Mainz, Germany. \\ E-mail: sebastian.seiffert@uni-mainz.de
}

Supporting Information

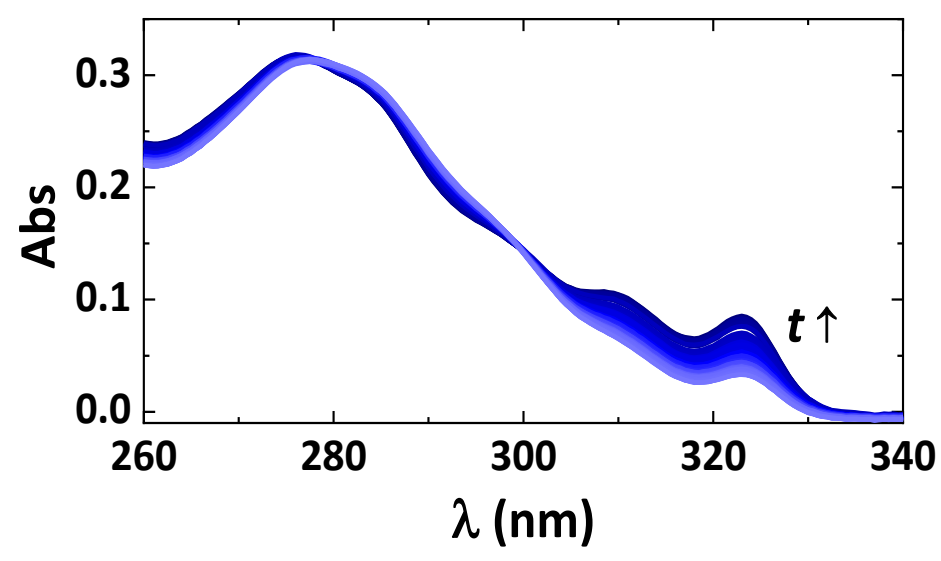

Figure S1. Time-dependent evolution of the zinc-terpyridine MLCT band, monitored via UV-vis spectroscopy during the complex formation process of zinc ions with tetra-arm PEG-terpyridine $\left(M_{\mathrm{w}}=10,000 \mathrm{~g} \mathrm{~mol}^{-1}\right)$, in presence of the additives EDTA and GDL. 


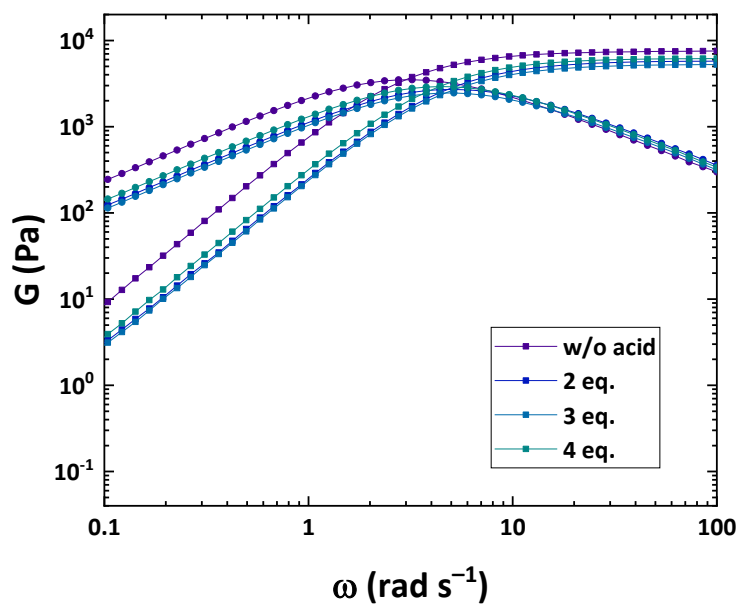

Figure S2. Comparison of the storage (filled circles) and loss (filled squares) moduli for zinc-tetra-arm PEG-terpyridine gels $\left(c=56 \mathrm{~g} \mathrm{~L}^{-1}\right)$ prepared by mixing two aqueous solutions of polymer and metal ions (purple) and gels prepared by a delayed gelation with the additive of 2 equivalents (dark blue), 3 equivalents (light blue), and 4 equivalents (green) of GDL.

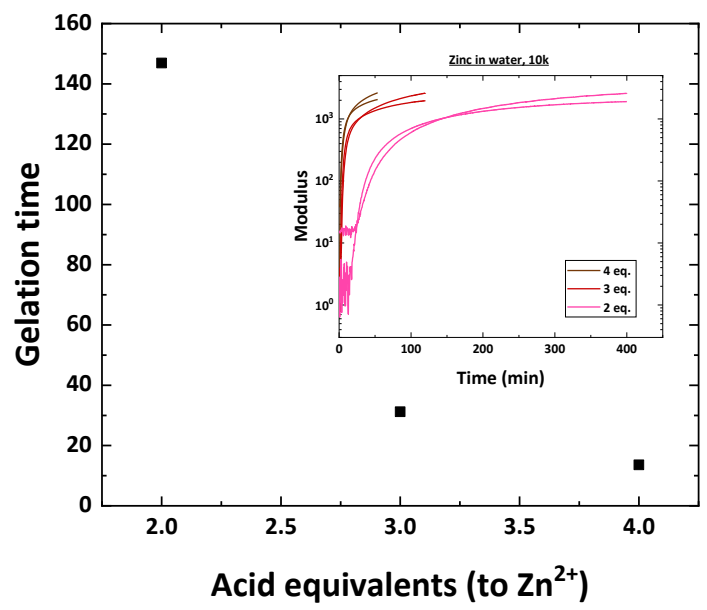

Figure S3. Gelation times depending on the amount of added GDL. Inset: Determination of the gelation time by rheology. The storage and loss moduli of three zinc tetra-arm PEG-terpyridine gels $\left(c=56 \mathrm{~g} \mathrm{~L}^{-1}\right)$ with different GDL content are plotted versus time (pink line: 2 eq. acid, red line: 3 eq. acid, and brown line: 4 eq. acid). 

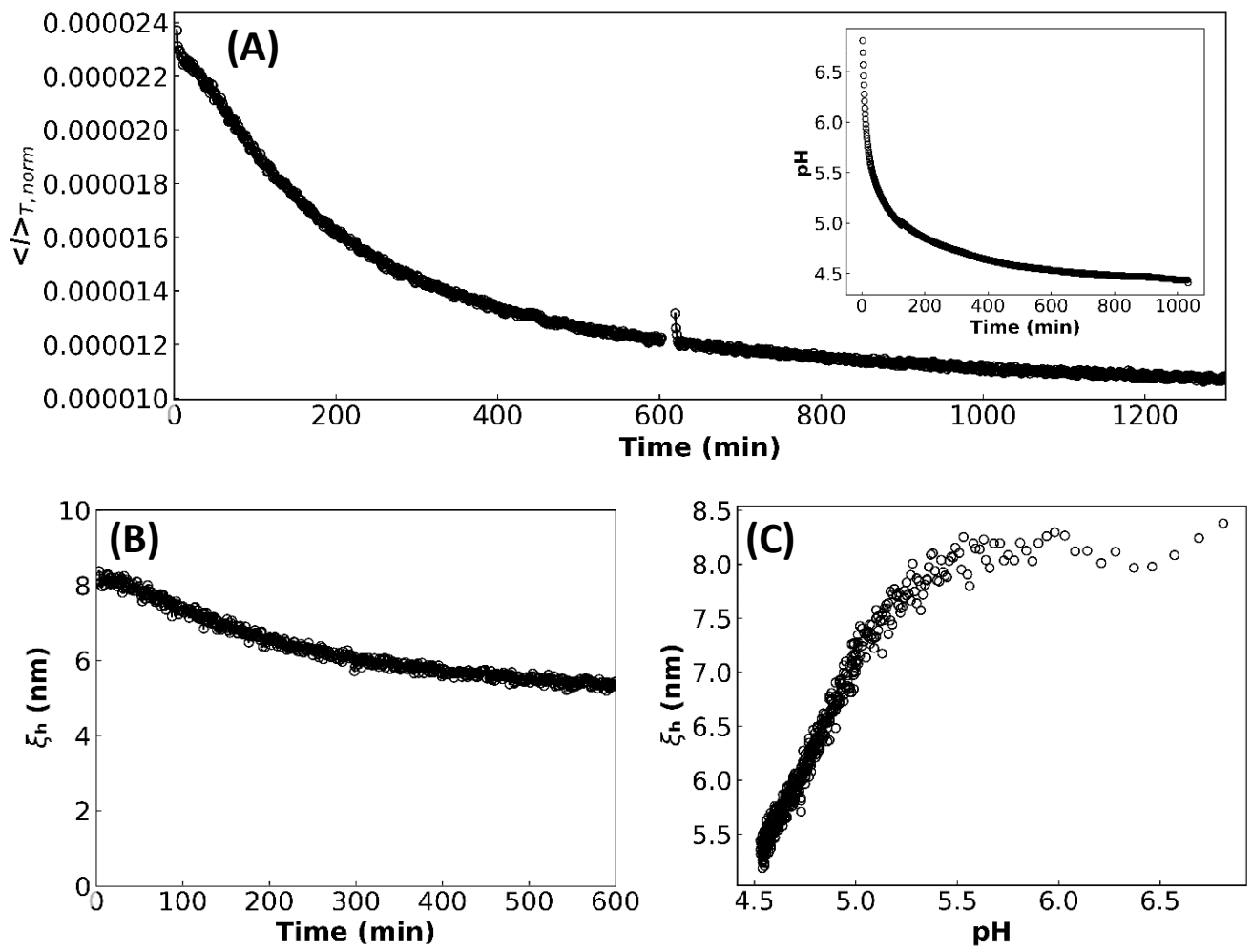

Figure S4. (A) Time-dependent normalized scattering intensity of an aqueous solution of tetra-arm PEG-terpyridine (56 g L-1) and GDL (1 eq. with respect to terpyridine groups). Inset: Evolution of pH over time due to hydrolysis of GDL. (B) The calculated hydrodynamic correlation length of the polymer is decreasing from $8 \mathrm{~nm}$ to $5.5 \mathrm{~nm}$ over time. (C) Correlation between the hydrodynamic correlation length and $\mathrm{pH}$. 

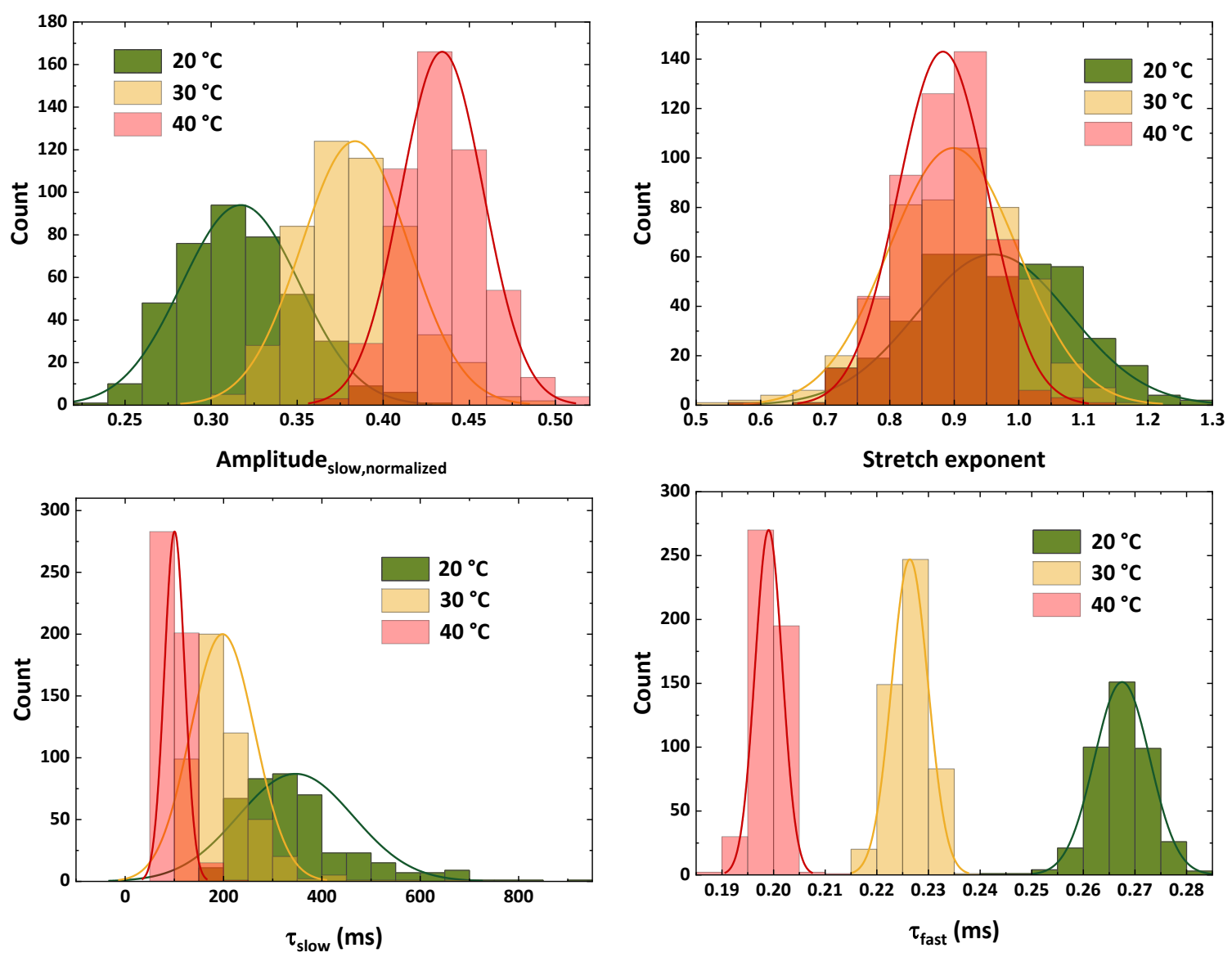

Figure S5. Intensity statistics (amplitude of the slow mode, stretch exponent $\alpha$, slow and fast relaxation time) of the tetraarm PEG-terpyridine zinc gel at $20^{\circ} \mathrm{C}$ (green), $30{ }^{\circ} \mathrm{C}$ (yellow), and $40{ }^{\circ} \mathrm{C}$ (red). All fit parameters exhibit a decrease in the distribution width with increasing temperature. The fast relaxation time $\tau$ fast is decreased with increasing temperature due to the enhanced thermal motion of polymer chains.

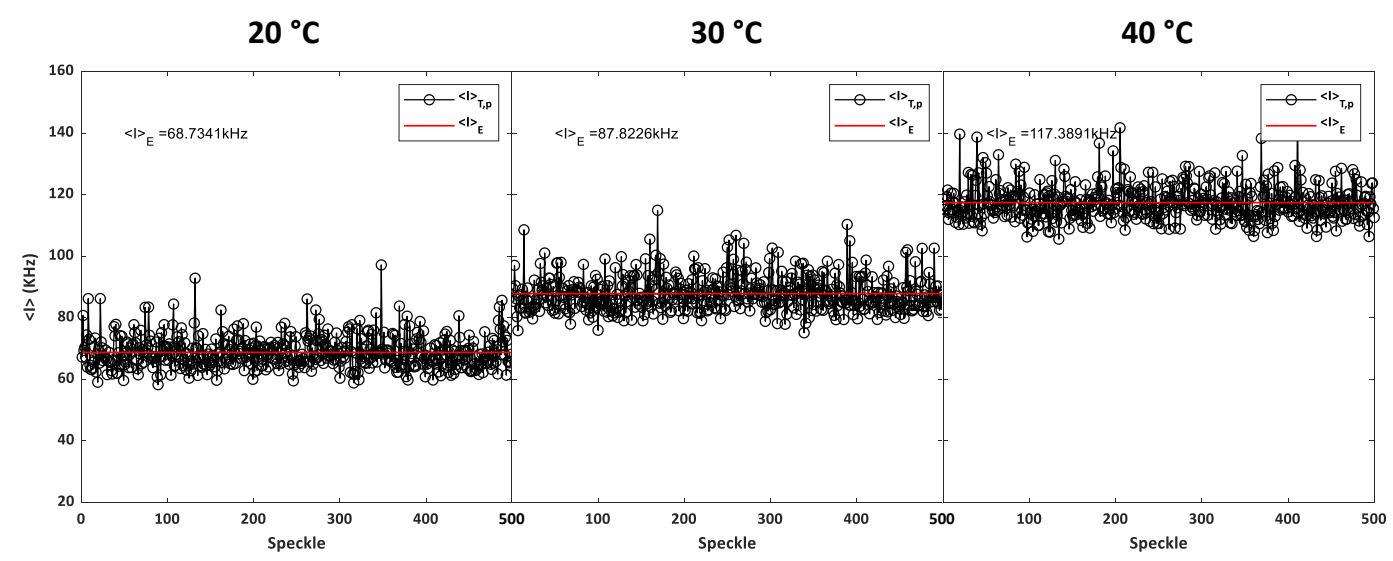

Figure S6. Recorded intensity speckles of the zinc tetra-arm PEG-terpyridine gel at temperatures of $20^{\circ} \mathrm{C}, 30^{\circ} \mathrm{C}$, and $40{ }^{\circ} \mathrm{C}$. 


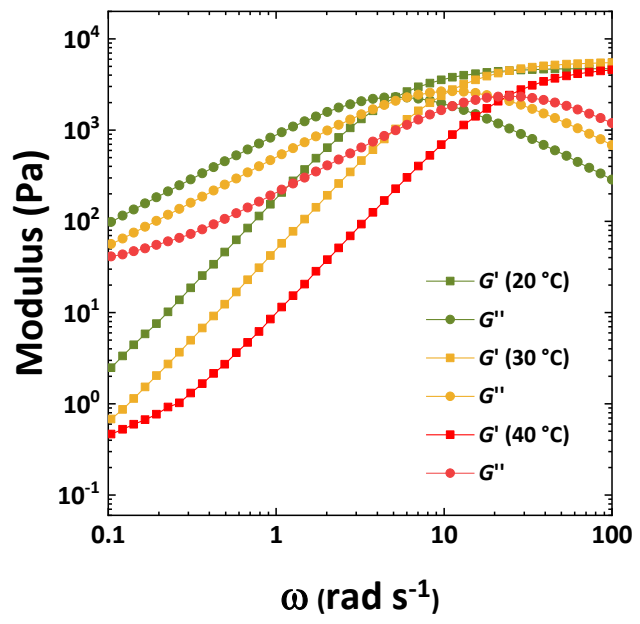

Figure S7. Frequency-dependent storage (full squares) and loss (full circles) moduli of a zinc tetra-arm PEG-terpyridine gel, probed at temperatures of $20^{\circ} \mathrm{C}$ (green), $30^{\circ} \mathrm{C}\left(\right.$ yellow), and $40^{\circ} \mathrm{C}($ red). 